\title{
The Pattern of Prevalence of Severe and Moderate Acute Malnutrition among Under-Five Children of Three Counties in a War-Torn Lakes State South Sudan
}

\author{
Article by Ebenezer O. Daniel ${ }^{1}$, Ademola M. Amosu ${ }^{2}$, Gabriel O. Ayeni ${ }^{3}$, Oladapo M. Olagbegi ${ }^{4}$ \\ 1, ${ }^{3}$ Texila American University Guyana \\ ${ }^{2}$ Babcock University Ilishan-Remo Nigeria \\ ${ }^{4}$ Rhodes University $S A$ \\ E-mail: dannypressy@yahoo.com ${ }^{1}$
}

\begin{abstract}
The prevalence of Severe Acute Malnutrition (SAM) and Moderate Acute Malnutrition (MAM) of under-five children, in south Sudan is reportedly high and is being aggravated by various negatives factors. The situation has attracted the attention of various international organizations like World Food Programme (WFP), United Nations Children Emergency Fund (UNICEF), and other International Nongovernmental Organizations (INGOs), for possible interventions. However, there are scarce study reports on the current prevalence pattern of SAM and MAM among under-five children, as it may assist in the implementation of nutrition therapy programme implemented by these humanitarian organizations.

A retrospective cohort study was conducted by collecting thirty six (36) month reported data of SAM and MAM of under-five children, from District Health Information Software (DHIS)-the standard source of health information in south Sudan, across three years; 2014, 2015 and 2016 for three counties, and was analyses using SPSS. Findings revealed statistical significant differences in SAM and MAM within each county and among the three counties across the three years. The results further revealed that there was rising median values of SAM and MAM among the counties across the three years. It was suggested that adequate pre-intervention survey should be conducted, reducing local government's sentiments and infiltration during screening exercise for malnutrition among under-five children, ensuring peace in south Sudan, and conduct of post-intervention surveys for feed-backs, may serve as a clue towards implementing an effective and efficient nutrition therapy programme in the war-torn Lakes State of south Sudan.
\end{abstract}

Keywords: Prevalence pattern, Malnutrition, SAM, MAM.

\section{Introduction}

In a developing country like south Sudan, various socio-economic problems are common, and this is always associated with negative effects like food scarcity with consequential malnutrition effect on the people especially the most vulnerable groups-women and the under-five children. According to World Food Program (2013), the situation in south Sudan is being worsened by the continuous insecurity following various internal conflicts which has caught the attention of various world-acclaimed donors and international Non-Governmental organizations (INGOs) The term "malnutrition" is actually a category of diseases that includes any illness resulting from an imbalance of calories or micronutrients (e.g. vitamins), either in dearth or excess. This study focused on the on the prevalence of Severe Acute Malnutrition (SAM) and Moderate Acute Malnutrition (MAM) in under-five children in Lakes state of South Sudan. The main purpose was to determine the pattern of prevalence of SAM and MAM in three counties, of Lakes state, where nutrition therapy program is being implemented.

The nutritional status of women and children is particularly important, because it is through women and their off-springs that the pernicious effects of malnutrition are propagated to future generations. A malnourished mother is likely to give birth to a low birth-weight (LBW) baby susceptible to disease and 
premature death, which only further undermines the economic development of the family and society, and continues the cycle of poverty and malnutrition. Although child malnutrition declined globally during the 1990s, with the prevalence of underweight children falling from $27 \%$ to $22 \%$ (Onis et al., 2004a), national levels of malnutrition still vary considerably (0\% in Australia; 49\% in Afghanistan)(WHO, 2003). The

largest decline in the level of child malnutrition was in eastern Asia where underweight levels decreased by one half between 1990 and 2000.

Underweight rates also declined in South-Eastern Asia (from 35\% to 27\%), and in Latin America and the Caribbean the rate of underweight children decreased by one third (from 9\% to 6\%) over the last 10 years. In contrast, South-Central Asia still has high levels of child malnutrition, even though the rate of underweight children declined from 50\% to $41 \%$ during the 1990s. In Africa, the number of underweight children actually increased between 1990 and 2000 (from 26 million to 32 million), and 25\% of all children under five years old are underweight, which signals that little changed from a decade earlier. The projection for 2005 is that the prevalence of child malnutrition will continue to decline in all regions in Africa, especially in sub-Saharan Africa (de Onis et al., 2004b). The World Food Program (WFP) estimates that 842 million people worldwide do not have access to enough food, approximately 146 million of which are children. Those included in this estimate range from simply too little food to extreme malnourishment. Severe Acute Malnutrition (SAM) and Moderate Acute Malnutrition (MAM) have been recognized as the most serious type of hunger, with 20 million and 35 million respective cases each year occurring in children less than 5 years of age (Edesia, 2012). According to WHO (2009), SAM is defined as a child having a weight-for-height ratio $>3$ standard deviations below the mean for their age, and MAM is a weight-for-height ratio $>2$ but $<3$ standard deviations. A notable point is that the majority of these cases occur in South Asia and Sub- Saharan Africa.

This study was therefore directed towards unraveling the actual prevalence pattern of acute malnutrition among under-five children, in order to give a clear direction for the nutrition therapy program, supported by WFP and UNICEF, for the vulnerable under-five children in three counties (Rumbek North, Yirol West, and Cueibet) of war-torn Lakes State of south Sudan

\section{Methodology}

Study Type: This study is a retrospective cohort study.

Study Setting: This study was conducted across three counties in Lakes state of south Sudan. They include Cueibet, Rumbek North and Yirol West counties.

Study Population: The study populations were the under-five children with either Severe Acute Malnutrition (SAM) or Moderate Acute Malnutrition (MAM), diagnosed by measuring Middle Upper Arm Circumference (MUAC) using MUAC tape.

Sampling Frame: The sampling frames for the study are the eight counties (8) counties in the Lakes state, one of the major ten states of south Sudan. This study focused on three counties where nutrition therapy programme for under-five children is being implemented.

Data collection: Data was collected from the District Health Information Software (DHIS) of the Republic of south Sudan. Thirty six (36) month data for each of the three counties across three years2014, 2015 and 2016 were obtained.

Data Analysis: Data analysis was done using the Statistical package for Social Science (SPSS) version 21. Friedman Analysis Of Variance (ANOVA) was used to compare SAM and MAM Middle Upper Arm circumference (MUAC) values of under-five Children across the three years in each of the three counties, while Wilconxon Signed Rank Test was used as post hoc analysis for Friedman ANOVA comparison of MUAC values for SAM and MAM across the three years. Kruskal Wallis was used to test for comparison of under-five children MUAC values for SAM and MAM across the three counties (in general), while Man Whitney U Test was used as the post hoc analysis for Kruskal Wallis of MUAC values for SAM and MAM of under-five children across the three counties in the three years- 2014, 2015 and 2016. Statistical level of significance was set at $\mathrm{P}$-value $<0.05$. 
Ethical considerations: An approval to conduct the study was obtained from Health Research Ethics Committee of the State Ministry of Health $(\mathrm{SMoH})$ of Lakes state in south Sudan, with endorsement of the Director General of the ministry.

\section{Results}

A total of 36 months data for SAM and MAM prevalence were extracted from the District Health Information Software (DHIS), for each of the three counties, across the three years-2014, 2015 and 2016.

Table 1. Friedman ANOVA comparison of SAM Middle Upper Arm circumference of Under Five Children across years 2014, 2015 and 2016 in each of the three counties

\begin{tabular}{|l|l|l|l|l|l|}
\hline Year & 2014 & 2015 & 2016 & & \\
\hline County & $\begin{array}{l}\text { Median } \\
\text { (MUAC) }\end{array}$ & $\begin{array}{l}\text { Median } \\
\text { (MUAC) }\end{array}$ & $\begin{array}{l}\text { Median } \\
\text { (MUAC) }\end{array}$ & $\begin{array}{l}\text { Chi- } \\
\text { Square }\end{array}$ & p-value \\
\hline Cueibet & 134.00 & 315.00 & 200.00 & 5.167 & 0.076 \\
\hline & & & & & \\
\hline Rumbek N. & 18.00 & 27.00 & 51.00 & 8.167 & $0.017^{*}$ \\
\hline & & & & & \\
\hline Yirol W. & 45.00 & 71.50 & 243.50 & 0.500 & 0.779 \\
\hline
\end{tabular}

*indicates significant difference at $\alpha=0.05$

The table above revealed a significant difference in SAM MUAC values of Rumbek North County only, across the three years. It also showed a rising median values across the three years in the three counties.

Table 2. Wilconxon Signed Rank Test post hoc analysis for Friedman ANOVA comparison for SAM MUAC across years 2014, 2015 and 2016

\begin{tabular}{|l|l|l|}
\hline County/Time Period & Chi-square & p-value \\
\hline Rumbek North & & \\
\hline 2014 vs 2015 & 0.863 & 0.388 \\
\hline 2014 vs 2016 & 2.511 & $0.012^{*}$ \\
\hline 2015 vs 2016 & 1.490 & 0.136 \\
\hline
\end{tabular}

*indicates significant difference at $\alpha=0.05$

The table above revealed that, the significant difference of SAM MUAC values in Rumbek North was between the 2014 and 2016.

Table 3. Friedman ANOVA comparison of MAM Middle Upper Arm circumference of Under Five Children across years 2014, 2015 and 2016 in each of the three counties

\begin{tabular}{|l|l|l|l|l|l|}
\hline Year & 2014 & 2015 & 2016 & & \\
\hline County & $\begin{array}{l}\text { Median } \\
\text { (MUAC) }\end{array}$ & $\begin{array}{l}\text { Median } \\
\text { (MUAC) }\end{array}$ & $\begin{array}{l}\text { Median } \\
\text { (MUAC) }\end{array}$ & $\begin{array}{l}\text { Chi-Square } \\
\text { p-value }\end{array}$ \\
\hline & 67.50 & 409.50 & 344.50 & 10.50 & $0.005^{*}$ \\
\hline Rumbek N. & 43.50 & 70.00 & 103.00 & 6.50 & $0.039^{*}$ \\
\hline & & & & & \\
\hline Yirol W. & 72.50 & 143.50 & 654.50 & 17.167 & $0.000^{*}$ \\
\hline
\end{tabular}

*indicates significant difference at $\alpha=0.05$ 
Texila International Journal of Public Health

Volume 5, Issue 3, Sep 2017

The table above showed significant differences in MAM MUAC values in each of the three counties across the three years. It also showed a rising median values in Rumbek North and Yirol west across the three years.

Table 4. Wilconxon Signed Rank Test post hoc analysis for Friedman ANOVA comparison for MAM MUAC across three years 2014, 2015 and 2016

\begin{tabular}{|l|l|l|}
\hline County/Time Period & $\begin{array}{l}\text { Chi- } \\
\text { square }\end{array}$ & P-value \\
\hline Cueibet & 1.465 & 0.075 \\
\hline 2014 vs 2015 & 2.353 & $0.019^{*}$ \\
\hline 2014 vs 2016 & -0.157 & 0.875 \\
\hline 2015 vs 2016 & 1.569 & 0.117 \\
\hline Rumbek North & 2.589 & $0.010^{*}$ \\
\hline 2014 vs 2015 & 1.020 & 0.308 \\
\hline 2014 vs 2016 & \multicolumn{2}{|}{} \\
\hline 2015 vs 2016 & \multicolumn{2}{|l|}{} \\
\hline Yirol West & 1.726 & 0.084 \\
\hline & 3.059 & $0.002^{*}$ \\
\hline 2014 vs 2015 & 2.981 & $0.003^{*}$ \\
\hline 2014 vs 2016
\end{tabular}

*indicates significant difference at $\alpha=0.05$

The table above revealed that, the significant difference in MAM MUAC values, across the three years in Cueibet, is between 2014 and 2016, in Rumbek North is between 2014 and 2016, and in Yirol West is both between 2014 and 2016, and between 2015 and 2016.

Table 5. Kruskal Wallis Test comparison of under-five children SAM and MAM MUAC across the three counties (In general)

\begin{tabular}{|l|l|l|l|l|l|}
\hline & \multicolumn{2}{l|}{ County } & & \\
\hline & Cueibet(n=36) & $\begin{array}{l}\text { Rumbek N. (n= } \\
36)\end{array}$ & $\begin{array}{l}\text { Yirol West (n } \\
=36)\end{array}$ & & \\
\hline $\begin{array}{l}\text { Malnutrition } \\
\text { type/Year }\end{array}$ & Median & Median & Median & H & p-value \\
\hline SAM 2014 & 134.00 & 18.00 & 45.00 & 12.933 & $0.002^{*}$ \\
\hline SAM 2015 & 315.00 & 27.00 & 71.00 & 25.891 & $0.001^{*}$ \\
\hline SAM 2016 & 200.00 & 51.00 & 243.50 & 19.344 & $0.001^{*}$ \\
\hline MAM 2014 & 67.50 & 43.50 & 72.50 & 3.090 & 0.213 \\
\hline MAM 2015 & 409.50 & 70.00 & 143.00 & 20.701 & $0.001^{*}$ \\
\hline MAM 2016 & 344.50 & 103.00 & 654.50 & 16.740 & $0.001^{*}$ \\
\hline
\end{tabular}

*indicates significant difference at $\alpha=0.05$

The table above showed that, there are significant differences in SAM MUAC values, among the three counties, across the three years-2014, 2015 and 2016. It also showed significant differences in MAM MUAC values, among the three counties in 2015 and 2016. 
Table 6. Man Whitney U Test post hoc analysis of SAM and MAM MUAC of under-five children across the three counties in 2014, 2015 and 2016

\begin{tabular}{|c|c|c|}
\hline County & Z score & P-value \\
\hline \multicolumn{3}{|l|}{ SAM 2014} \\
\hline Cueibet vs Rumbek North & -2.890 & $0.004 *$ \\
\hline Cueibet vs Yirol West & -1.559 & 0.119 \\
\hline Rumbek North vs Yirol West & -3.062 & $0.002 *$ \\
\hline \multicolumn{3}{|l|}{ SAM 2015} \\
\hline Cueibet vs Rumbek North & -4.158 & $0.000^{*}$ \\
\hline Cueibet vsYirol West & -4.042 & $0.000^{*}$ \\
\hline Rumbek North vs Yirol West & -2.599 & $0.009 *$ \\
\hline \multicolumn{3}{|l|}{ SAM 2016} \\
\hline Cueibet vs Rumbek North & -3.984 & $0.000^{*}$ \\
\hline Cueibet vs Yirol West & -.404 & 0.686 \\
\hline Rumbek North vs Yirol West & -3.580 & $0.000^{*}$ \\
\hline \multicolumn{3}{|l|}{ MAM 2015} \\
\hline Cueibet vs Rumbek North & -3.811 & $0.000^{*}$ \\
\hline Cueibet vs Yirol West & -3.522 & $0.000^{*}$ \\
\hline Rumbek North vs Yirol West & -2.225 & $0.026^{*}$ \\
\hline \multicolumn{3}{|l|}{ MAM 2016} \\
\hline Cueibet vs Rumbek North & -2.656 & $0.008^{*}$ \\
\hline Cueibet vs Yirol West & -2.367 & $0.018 *$ \\
\hline Rumbek North vs Yirol West & -3.580 & $0.000 *$ \\
\hline
\end{tabular}

*indicates significant difference at $\alpha=0.05$

The table above revealed that the existing significant differences of SAM MUAC in under-five children, are among the three possible combinations of the three counties (Cueibet vs Rumbek North, Cueibet vs Yirol West, and Rumbek North vs. Yirol West) across three years except in 2014 SAM MUAC and 2016 SAM MUAC between Cueibet and Yirol West. It also showed significant differences in MAM MUAC among the three possible combinations of the counties, across 2015 and 2016.

\section{Discussion}

This study aimed to determine the pattern of prevalence of Severe Acute Malnutrition (SAM) and Moderate Acute Malnutrition (MAM) in three counties of war-torn Lakes State of south Sudan. In the results, and considering each county, there was a statistical significant difference in SAM prevalence of Rumbek North County only, across the three years 2014, 2015 and 2016. It was also discovered that, there was a rising median values of SAM across the three years in the three counties. The post-hoc analysis further revealed that the statistical significant difference of SAM prevalence in Rumbek North was between the 2014 and 2016.

The possible reasons for these significant differences, in conjunction with rising median values of SAM prevalence (poor outcome) across the three counties, were as suggested by the report of a similar study (Mwanza et al, 2016) for SAM cases of under-five children, in three districts of eastern province in Zambia. These include; dependence on donor-funds for feeding, and consistent stock outs of food provision by humanitarian organizations, both of which are applicable in the three counties under this study.

Taking the MAM prevalence into consideration, the result revealed statistical significant differences in each of the three counties across the three years. It also showed a rising median values of MAM 
prevalence (poor outcome) in Rumbek North and Yirol west across the three years. The result of the posthoc analysis further revealed that, the significant difference in MAM prevalence, across the three years, in Cueibet and Rumbek North is between 2014 and 2016, while in Yirol west are both between 2014 and 2016, and between 2015 and 2016. This may be attributed to a possible reason, as suggested by Neitzel (2011) in a study conducted in five regions in Ethiopia (Tigray, Afar, Amhara, Oromia and SNNPR). The study suggested food insecurity as an implicating factor determining the prevalence of MAM among under-five children, which is closely applicable in the context of this study, as a possible reason.

Considering the relationship among the three counties, the results of this study showed that, there are significant differences in SAM prevalence, among the three counties, across the three years-2014, 2015 and 2016. It also showed significant differences in MAM prevalence, among the three counties in 2015 and 2016. The post-hoc analysis further revealed that, the existing significant differences of SAM prevalence in under-five children, are among the three possible combinations of the three counties (Cueibet vs Rumbek North, Cueibet vs Yirol West, and Rumbek North vs. Yirol West) across three years except in 2014 SAM and 2016 SAM between Cueibet and Yirol West.

The results of this study also showed statistical significant differences in MAM prevalence among the three possible combinations of the counties, across 2015 and 2016. The significant differences in SAM prevalence among the three counties, in the three possible combinations, across the three years, and MAM prevalence among the three possible combinations, among the three counties, across 2015 and 2016, may not be unconnected with the possibility that, there may be variations in diagnosis according to standard by the Community Nutrition Volunteers (CNVs) and Community Health Workers (CHWs) in each county using MUAC tapes, and political sentiments in screening exercise, which may be twisted, in collaborations with health workers, in any county, hence influencing the wrong diagnosis of children, (increasing the false positives) in order to attract more nutrition intervention funds, may also be responsible for these statistical significant differences.

\section{Conclusion}

The results obtained from this study do not support the null hypotheses of the study. The first null hypothesis states that, there will be no significant differences in the prevalence of SAM and MAM among under-five children in each of the three counties, across the three years in Lakes State of south Sudan. The results of this study showed conspicuous significant differences in SAM and MAM prevalence in each county across the years. A major factor recognized for the significant differences in SAM and MAM values, being an increase in food insecurity in south sudan. The second null hypothesis which states that, there will be no significant differences in SAM and MAM prevalence among under-five children in the three counties, across the three years in Lakes State of south Sudan, was also at variance with the results of this study, as there were various significant differences in SAM and MAM prevalence among the three counties, across the three years. The possibility of variations in diagnosis according to standard by Community Nutrition Volunteers (CNVs) and Community Health Worhers (CHWs) in each county using MUAC tapes, and political sentiments during screening for malnutrition among under-five children, were suggested as possible reasons for these significant differences.

In overall, this study has shown that, there are variations in the pattern of prevalence of acute malnutrition among under-five children, within and among three counties, across three years. Hence it is a pointer to the fact that there is a need for a careful determination of the pattern of prevalence of Severe Acute Malnutrition (SAM) and Moderate Acute Malnutrition (MAM), as peculiar to each county. This will serve as a clue to how nutrition therapy programme in each county will be implemented, monitored, and evaluated. There is a need for the donors (like WHO and UNICEF) and the implementing partners like International Non-Governmental Organizations (INGOs) to conduct pre-intervention surveys to determine the peculiarity of the prevalence of acute malnutrition, among under-five children in each county. The conduct of the and post-intervention evaluations will therefore serve as a feedback of interventions for each county, and suggest possible ways forward on the best method to be adopted in the 
nutrition therapy programme in various regions where it is being implemented. The government (especially at the local levels) should also be made to conform to the standard protocols guiding the screening of malnourished children in order to have a true picture of the situation before an appropriate intervention will be enacted. Re-enforcing every possible means to ensure a peaceful co-existence among the citizens of south Sudan will also play a pivotal role in reducing the negative prevalence pattern of acute malnutrition among these vulnerable group of the population-the under-five children.

\section{References}

[1]. Abdalla MA, Saad A, Abdullahi HE, Abdul Gader H (2009). Socio-economic aspects influencing food consumption patterns among children under age of five in rural area of Sudan. Pak. J. Nutr. 8:653659.

[2]. Adair, L. S., Fall, C. H., Osmond, C., Stein, A. D., Martorell, R., Ramirez-Zea, M., Sachdev, H. S., Dahly, D. L., Bas, I., Norris, S. A., Micklesfield, L., Hallal, P. \& Victora, C. G. (2013): Associations of linear growth and relative weight gain during early life with adult health and human capital in countries of low and middle income: findings from five birth cohort studies. Lancet, 382, 525-34.

[3]. Agozie C Ubesie, N. S. ( 2012). Malnutrition Admitted at the University of In Nigeria Teaching HOSPITAL, Enugu: 10years retrospective review. . NutritionJournal, 1.

[4]. Ahmed, F. .. (2014). Effects of malnutrition under five years of age and its complications. Carechallenge.

[5]. Ahmed, T., Auble, D., Berkley, J.A., Black, R., Ahern, P.P., Hossain, M., Hsieh, A., Ireen, S., Arabi, M., Gordon, J.I. (2014). An evolving perspective about the origins of childhood under nutrition and nutritional interventions that includes the gut microbiome. Annals of the New York Academy of Sciences. 1749-6632. http: //dx.do i.org/10. 1111/nya s.12487

[6]. Amy L. Rice, L. S. (2000). Malnutrition as an underlying cause of childhood deaths associated with infectious diseases in developing countries. Bulletin of the World Health Organisatioon.

[7]. Ashworth, A.; Ferguson, E. (2009). "Dietary Counselling in the Management of Moderate Malnourishment in Children." Food and Nutrition Bulletin.2009, 30(Suppl. 3):S405-S433Bachmann.

[8]. Black RE, Allen LH, Bhutta ZA, Caulfield LE, Onis M, Ezzati M, Mathers C, Rivera J (2008). Maternal and child under nutrition: global and regional exposures and health consequences. Lancet 371:243-260.

[9]. Checchi, F. \& Robinson, W. C. (2013). Mortality among populations of southern and central Somalia affected by severe food insecurity and famine during 2010-2012. Rome, Washington.

[10].De-Onis M, Blössner M, Borghi E, Morris R, Frongillo E (2004a). Methodology for estimating regional and global trends of child malnutrition. International Journal of Epidemiology (in press).

[11].Lehmann A.B. (1991). Nutrition in old age: an update and questions for future research: part I. Reviews in Clinical Gerontology 1, 135-145.

[12].Mwanza, K.M, Okop J, Puoane T (2016): Evaluation of outpatient therapeutic programme for management of severe acute malnutrition in three districts of the eastern province, Zambia.

[13].Neitzel J (2011). The targeted supplementary feeding programme.

[14].Stephan LS, Lathum MC, Ohesen EA (2000).Global malnutrition. Parasitology 121:55-22.

[15].UNICEF (2014). Chief of Strategic Communication, UNICEF South Sudan. www.unicef.org

[16].UNICEF, WHO \& World-Bank (2012).UNICEF-WHO-World Bank Joint Child Malnutrition Estimates. (UNICEF, New York; WHO, Geneva; the World Bank, Washington, DC).

[17].VOA (2016). Child malnutrition crisis deepens in South Sudan http://www.voanews.com/a/childmalnutrition-crisis-deepens-in-south-sudan/3451608.html

[18].WFP (2015). UNICEF And WFP resolve to defeat malnutrition in South Sudan

[19]. WHO (2000) World Health Organization, Dept of Nutrition for Health and Development. Nutrition for health and development: A global agenda for combating malnutrition. WHO

[20].WHO, (2003) “The Management of Nutrition in Major Emergencies" 
Texila International Journal of Public Health

Volume 5, Issue 3, Sep 2017

[21].WHO 2013: Guideline update: Technical aspects of the management of severe acute malnutrition in infants and children. Geneva: World Health Organization; 2013.

[22].WHO and UNICEF. 2009. WHO child growth standards and the identification of severe acute malnutrition in infants and children: A Joint Statement by the World Health Organization and the United Nations Children's Fund. Geneva: WHO.

[23].WHO, (2003) "The Management of Nutrition in Major Emergencies"

[24].WHO, WFP, UN/SCN, UNICEF. (2007). Community-Based Management of Severe Acute Malnutrition. A Joint Statement by the World Health Organization, the World Food Programme, the United Nations System Standing Committee on Nutrition and the United Nations Children's Fund (UNICEF). New York: UNICEF. May 2007.

[25].WHO. (2012). Technical Note: Supplementary Foods for the Management of Moderate Acute Malnutrition in Infants and Children 6-59 Months of Age. Geneva: WHO. 